\title{
Pratiques d'élevage de porcs au Sud-Bénin : utilisation des plantes dans la gestion alimentaire et sanitaire
}

\author{
OHOUKO Okri Fréjus Hans ${ }^{1,2 *}$, KOUDOUVO Koffi ${ }^{2}$, DOUGNON Tossou Jacques ${ }^{1}$, \\ AGBONON Amegnona ${ }^{2}$, GBEASSOR Messanvi ${ }^{2}$ \\ ${ }^{1}$ Unité de Recherches en Microbiologie Appliquée et Pharmacologie des Substances Naturelles, Laboratoire de Recherche en Biologie \\ Appliquée, Département de Production et Santé Animales, Ecole Polytechnique d'Abomey-Calavi, Université d'Abomey-Calavi, \\ 01BP2009 Cotonou, Bénin \\ ${ }^{2}$ Laboratoire de Physiologie et Pharmacologie des Substances Naturelles, Centre de Recherches et de Formation sur les plantes \\ Médicinales, Faculté des Sciences, Université de Lomé, 01BP1515 Lomé, Togo \\ *Auteur correspondantEmail:ohonkofrjus@yahoo.comou ohonkofrjus@,e-urmapha.com; Tél: +229 64301284
}

Mots clés : Porc, système d'élevage, plantes, Sud-Bénin

Keywords: Pigs, breeding system, plants, South of Benin.

Publication date 30/06/2020, http://m.elewa.org/Journals/about-japs/

\section{RESUME}

L'élevage de porcs au Bénin continue de faire face aux contraintes d'ordres alimentaires et sanitaires, caractéristiques fondamentales de la régression du taux de production porcine. L'objectif de cette étude est de répertorier les pratiques relatives à l'utilisation des plantes pour des fins alimentaires et de santé animales en vue de les améliorer. Ainsi, 70 éleveurs de porcs ont été interviewés sur la base d'un questionnaire prenant en compte les techniques d'élevages, la gestion sanitaire, alimentaire et des infrastructures, mais aussi l'utilisation des plantes dans l'élevage. Il en ressort que la majorité des éleveurs sont de sexe masculin $(91,43 \%)$ et essentiellement caractérisés par les niveaux d'études primaire $(34,29 \%)$ et secondaire $(40 \%)$. Les porcs de races améliorées $(94,29 \%)$ sont priorisés au Sud-Bénin et ces porcs sont élevés en claustration permanente pour la plupart avec une dominance d'habitats en dur $(85,71 \%)$. La majorité des éleveurs formulent eux même leurs rations alimentaires avec des suppléments de fourrages. La gale $(68,57 \%)$ et la peste porcine africaine $(38,57 \%)$ ont été les pathologies les plus citées par les éleveurs. Au total, 42 espèces végétales appartenant à 23 familles botaniques ont été identifiées au cours de cette étude. Ces plantes sont utilisées aussi bien pour l'alimentation $(60 \%)$ que pour le traitement de maladies $(19 \%)$ ou les deux modes (29\%). Moringa oleifera (15,14\%), Carica papaya (13,3\%), Manihot esculenta (12,39\%), Ipomea aquatica $(9,17 \%)$ et Talinum triangulare $(8,72 \%)$ ont été les plantes les plus citées. Les feuilles (78\%) constituent la partie la plus utilisée au niveau des plantes. Au total, 22 recettes ont été recensées, constituées d'une seule plante chacune et administrées le plus par voie orale et sous la forme de crudité. La recette impliquant Carica papaya $(17,44 \%)$ dans le traitement de la parasitose digestive a été plus citée. De plus, une indication a été enregistrée auprès des éleveurs de porcs du Sud-Bénin pour le traitement de la peste porcine africaine impliquant Cochlospermum planchonii (6,98\%). Les résultats obtenus sont la preuve que la flore béninoise dispose de plantes utilisées en alimentation et dans les soins de santé porcine qui peuvent faire objet de la production de provende et de phytomédicament utilisables en médecine vétérinaire. 


\begin{abstract}
Pig farming in Benin continues to face feed and health constraints leading to the decline in the rate of pig production. This study aimed to identify practices relating to the use of plants for feeding and animal health purposes in order to improve pig production. It appears that the respondents were predominantly male $(91.43 \%)$ and characterized by primary $(34.29 \%)$ and secondary (40\%) education levels. Improved breed pigs were more prevalent in Southern Benin (94.29\%) and most of the pigs are kept in permanent confinement with dominance of permanent habitats $(85.71 \%)(P<0.001)$. The majority of breeders formulate their own feeds with forage supplements. Scabies (68.57\%) and African swine fever (38.57\%) are the major pathologies reported by farmers.Forty two (42) plant species belonging to 23 botanical families have been identified during this study. These plants are used for either food (60\%), disease treatment $(19 \%)$ or both $(29 \%)$. Moringa oleifera $(15.14 \%)$, Carica papaya $(13.3 \%)$, Manihot esculenta (12.39\%), Ipomea aquatica $(9.17 \%)$ and Talinum triangulare $(8.72 \%)$ are the most commonly mentioned species. Leaves $(\mathbf{7 8 \%})$ are the most commonly parts of plant used in this study area. In total, 22 recipes were recorded and each constituted of a single plant and are mostly administered orally (95.45\%) and frequently in the raw form (77.27\%). Recipe involving Carica papaya (17.44\%) in the treatment of digestive parasitosis was more recorded. In addition, only one indication was recorded for ASF involving Cochlospermum planchonii, cited by farmers in Southern Benin ( 6.98\%). The results obtained prove that the Benin flora has plants used in pig feeding and health care which can be subject to the production of feed and phytomedicine for veterinary medicine use.
\end{abstract}

\section{INTRODUCTION}

La production porcine contribue à $1,4 \%$ de la production animale en Afrique de l'Ouest (FAOSTAT, 2019). Elle constitue une activité secondaire procurant de revenus supplémentaires à une catégorie de couches socio-professionnelles de la population d'Afrique (Agbokounou et al., 2016). Au Bénin, la filière porcine constitue un secteur d'activité génératrice de revenus pour bon nombre d'acteurs du domaine avec une production évaluée à 504000 têtes de porcs en 2018 (FAOSTAT, 2020). Dans le soucis d'améliorer la filière au profit des éleveurs et par conséquent de tous les acteurs de la chaîne de production, des recherches ont été effectuées et se sont axées sur l'amélioration des performances de production et de reproduction des porcs (Youssao et al., 2008a; Youssao et al., 2008b; Youssao et al., 2009;

\section{METHODOLOGIE}

3.1 Zone de l'étude : Elle a pris en compte les départements de l'Atlantique situés entre $6^{\circ}$ $40^{\prime} \mathrm{N}$ et $2^{\circ} 30^{\prime} \mathrm{E}$ et l'Ouémé situé entre $6^{\circ} 37^{\prime} \mathrm{N}$ et $2^{\circ} 31^{\prime} \mathrm{E}$. Ils sont constitués respectivement de
Dotché et al., 2018), l'alimentation des porcs à travers la valorisation des ressources fourragères et non conventionnelles (Codjo, 2003; Hedji et al., 2015; Agbokounou et al., 2016; Kiki et al., 2018). Cependant, des contraintes d'ordre alimentaires et sanitaires continuent de freiner l'expansion de la filière porcine en Afrique de l'Ouest en général et au Bénin en particulier. Dans ce contexte, des défis restent à relever pour rehausser la production animale et participer ainsi à la sécurité alimentaire, l'un des piliers du développement durable en Afrique. L'objectif général de cette étude est d'évaluer les pratiques d'élevage de porcs au Sud-Bénin. Il s'agit de répertorier les pratiques relatives à l'utilisation des plantes pour des fins alimentaires et de santé animales en vue de les améliorer. .

huit et neuf communes respectivement. Les communes de Sèmè-Kpodji, Porto-Novo et Adjarra dans l'Ouémé et celle d'Abomey-Calavi dans l'Atlantique ont été parcourues (figure 1). 
Les climats sont de type subéquatorial avec quatre saisons, deux pluvieuses (une grande d'avril à juillet et une petite d'octobre à novembre) et deux sèches (une grande de décembre à mars et une petite d'août à septembre). Ils bénéficient par an d'une pluviométrie comprise entre $900 \mathrm{~mm}$ et $1500 \mathrm{~mm}$ avec une température variant de 25 à $30^{\circ} \mathrm{C}$ (Kiki et al., 2018). Les principales cultures sont le maïs, le manioc, l'arachide, le palmier à huile, les cultures maraîchères et le niébé. L'élevage comprend principalement les bovins surtout dans l'Atlantique, la pisciculture, le petit élevage et l'élevage de porcs (INSAE, 2015).

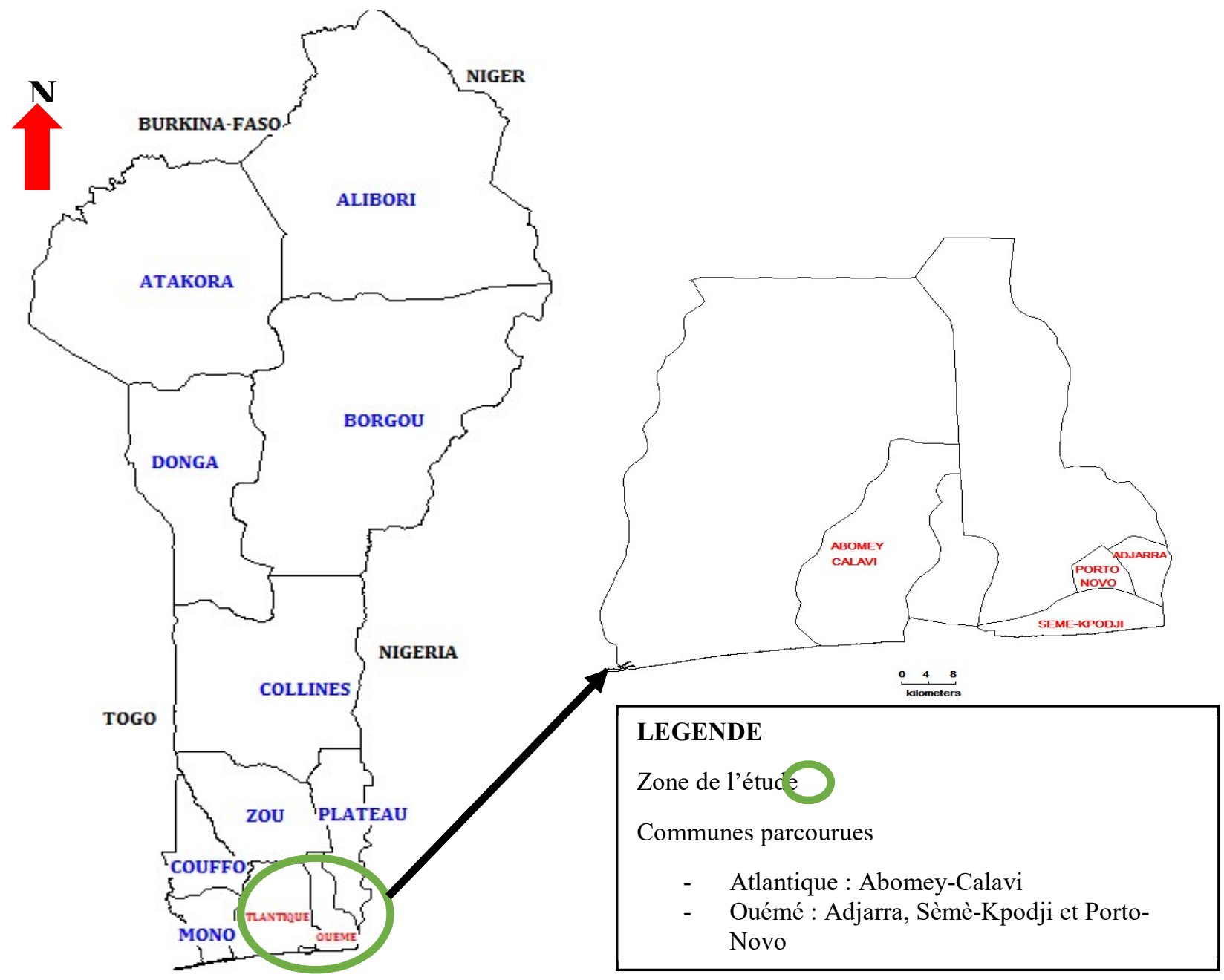

Conception : OHOUKO Fréjus, Mars 2020

Figure 1 : Zone d'étude sur les pratiques d'élevages de porcs au Sud-Bénin

3.2 Collecte des données et traitements : L'enquête a consisté en une interview semistructurée avec les éleveurs sur la base d'un questionnaire (Koudouvo et al., 2011). Au total, 70 éleveurs de porcs ont été enquêtés au Sud du Bénin. Les éleveurs ont été choisis suivant les critères d'accessibilité à leur porcherie (Kiki et al.,
2018). Ici, ces critères sont définis par l'acceptabilité de l'éleveur à répondre au questionnaire et sa disponibilité. Le questionnaire renseigne sur l'identité de l'informateur, la race de porc élevée, la gestion de l'alimentation, des infrastructures et celle sanitaire, le mode d'élevage, l'usage de plantes 
dans l'élevage et le but d'utilisation, les pathologies porcines rencontrées chez les animaux, les remèdes traditionnels à base de plantes utilisés pour les traiter (plantes, organes utilisés, modes de préparation, posologies et voies d'administration). Après l'interview avec les éleveurs, les plantes recensées en langues locales ont été identifiées à partir des documents de références de Adjanohoun (1989); EkluNatey et Balet (2012) et confirmé par l'équipe du Professeur Koffi AKPAGANA. Ensuite, les familles de ces plantes dont les noms scientifiques ont été identifiés ont été recherchées dans la flore analytique du Bénin de Akoègninou et al. (2006). La fréquence de citation des plantes a été calculée par la formule :

\section{RESULTATS}

Les résultats enregistrés prennent en compte les caractéristiques socio-professionnelles des éleveurs enquêtés, le mode de l'élevage, la gestion sanitaire de l'élevage des porcs ainsi que l'usage des plantes dans les élevages.

\subsection{Caractéristiques socio-} professionnelles des éleveurs et mode d'élevage de porcs au Sud-Bénin: Les éleveurs de porcs enquêtés sont majoritairement des hommes $(91,43 \%)$ âgés en moyenne de 44,24 ans. Ils ont le niveau d'étude primaire $(34,29 \%)$ et secondaire (40\%). Toutefois, $10 \%$ des éleveurs de porcs enquêtés sont caractérisés par un niveau d'étude universitaire. L'agriculture et l'artisanat constituent les activités principales des éleveurs de porcs. Seulement 15,71 \% des enquêtés mènent l'élevage de porcs comme activité principale (Tableau 1). La taille de cheptel moyenne était de 18,41 porcs. Les enquêtés élèvent plus $(\mathrm{p}<0,001)$ les porcs de race améliorée $(94,29 \%$ ) que les porcs de race locale
$\mathbf{F c}=\frac{\mathbf{n}}{\mathbf{N}} \times 100$

Fc: Fréquence de citation; $\mathrm{n}$ : Nombre de citations d'une plante; $\mathrm{N}$ : Nombre totale de plantes

3.3 Analyses statistiques: Les données collectées ont été encodées dans le logiciel Excel 2013 et analysées avec le logiciel SAS (SAS, Cary, NC, 2006).Les fréquences ont été calculées par la procédure Procfreq du SAS. Le test de Chi carré a permis de comparer les fréquences. Pour chaque fréquence, un intervalle de confiance (IC) à $95 \%$ a été calculé selon la formule :

$\mathrm{IC}=\mathrm{P} \pm 1,96 \sqrt{\frac{P(1-P)}{N}}$

où $\mathrm{P}$ est la fréquence relative et $\mathrm{N}$ la taille de l'échantillon

$(5,71 \%)$. Le mode d'élevage le plus fréquent est la claustration permanente $(p<0,001)$ dans des habitats en dur, bois et en terre battue. Le pourcentage d'élevage avec des habitats en dur $(85,71 \%)$ a été significativement supérieur $(\mathrm{p}<0,001)$ à ceux des élevages avec des habitats en terre battue $(8,57 \%)$ et en bois $(5,71 \%)$. Les porcs à de différents stades de production (truies gestantes, porcelets en engraissement, truies vides) sont disposés pour la plupart dans des loges individuelles $(90 \%)$ au sein d'un même bâtiment. Les porcs étaient nourris en majorité $(92,86 \%)$ par des aliments constitués d'un mélange au hasard de deux ou plusieurs matières premières. Les matières premières utilisées dans les mélanges sont présentées dans la figure 2 . Elles sont dominées par le tourteau de palmiste $(80 \%)$, le son de maïs $(53 \%)$, le son de blé $(51 \%)$, le son de soja $(33 \%)$ et les épluchures et tubercules de manioc $(31 \%)$. 
Tableau 1 : Caractéristiques socio-professionnelles des éleveurs et mode d'élevage de porcs au SudBénin

\begin{tabular}{|c|c|c|}
\hline Variables & $\begin{array}{c}\text { Fréquences } \pm \text { Intervalle } \\
\text { de confiance }(\%) \\
(\mathrm{N}=70)\end{array}$ & $\begin{array}{c}\text { Test de Chi } \\
\text { carré }\end{array}$ \\
\hline \multicolumn{3}{|l|}{ Sexe } \\
\hline Masculin & $91,43^{a} \pm 6,6$ & \multirow{2}{*}{$* * *$} \\
\hline Féminin & $8,57^{\mathrm{b}} \pm 6,6$ & \\
\hline \multicolumn{3}{|l|}{ Niveau d'étude } \\
\hline Universitaire & $10^{\mathrm{b}} \pm 7$ & \multirow{4}{*}{$* *$} \\
\hline Non scolarisé & $15,71^{\mathrm{b}} \pm 8,5$ & \\
\hline Primaire & $34,29^{a} \pm 11,1$ & \\
\hline Secondaire & $40^{a} \pm 11,5$ & \\
\hline \multicolumn{3}{|l|}{ Activités principales } \\
\hline Artisanat & $28,57^{a} \pm 10,6$ & \multirow{5}{*}{$* *$} \\
\hline Agriculture & $27,14^{a} \pm 10,4$ & \\
\hline Elevage & $15,71^{\mathrm{b}} \pm 8,5$ & \\
\hline Commerce & $11,43^{\mathrm{b}} \pm 7,5$ & \\
\hline Autres & $17,15^{\mathrm{b}} \pm 8,8$ & \\
\hline \multicolumn{3}{|l|}{ Races } \\
\hline Améliorée & $94,29^{\mathrm{a}} \pm 5,4$ & \multirow[t]{2}{*}{ *** } \\
\hline Locale & $5,71^{\mathrm{b}} \pm 5,4$ & \\
\hline \multicolumn{3}{|l|}{ Mode d'élevage } \\
\hline Claustration permanente & $98,57^{\mathrm{a}} \pm 2,8$ & \multirow[t]{2}{*}{$* * *$} \\
\hline Claustration temporaire & $1,43^{\mathrm{b}} \pm 2,8$ & \\
\hline \multicolumn{3}{|l|}{ Types d'habitats } \\
\hline Dur & $85,71^{\mathrm{a}} \pm 8,2$ & \multirow{3}{*}{$* * *$} \\
\hline Bois & $5,71^{\mathrm{b}} \pm 5,4$ & \\
\hline Terre battue & $8,57^{\mathrm{b}} \pm 6,6$ & \\
\hline \multicolumn{3}{|l|}{ Loges } \\
\hline Combinée & $5,71^{\mathrm{b}} \pm 5,4$ & \multirow{3}{*}{$* * *$} \\
\hline Individuelles & $90^{a} \pm 7$ & \\
\hline Séparées & $4,29^{\mathrm{b}} \pm 4,7$ & \\
\hline \multicolumn{3}{|l|}{ Types de ration } \\
\hline Aliment commercial & $7,14^{\mathrm{b}} \pm 6$ & \multirow{2}{*}{$* * *$} \\
\hline Mélange de 02 ou plusieurs matières premières & $92,86^{\mathrm{a}} \pm 6$ & \\
\hline
\end{tabular}

NS : non significatif, $*: \mathrm{P}<0,05 ; * *: \mathrm{P}<0,01 ; * * *: \mathrm{P}<0,001 ; \mathrm{N}:$ Nombre d'éleveurs enquêtés. les fréquences intra classes de la même colonne suivies de lettres $(a, b)$ différentes présentent une différence significative au seuil de 5\% 


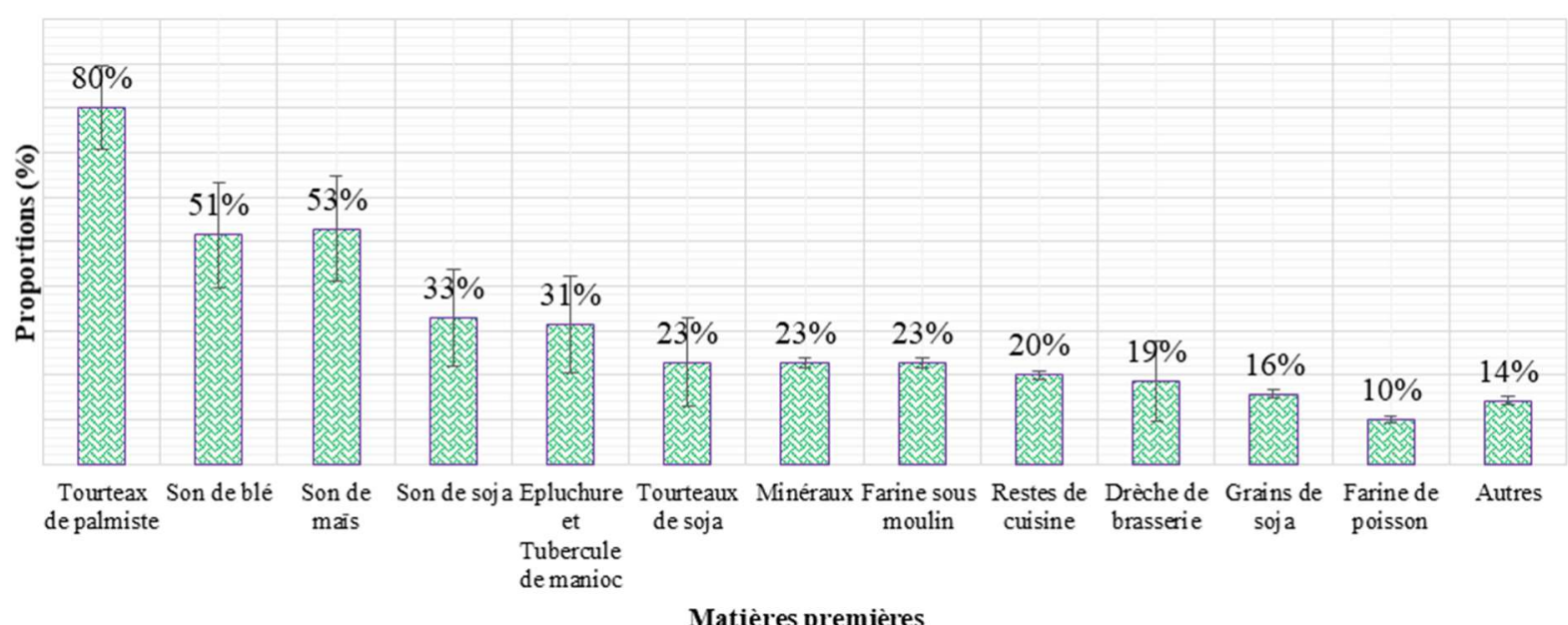

Minéraux : sel de cuisine, coquille d'huitre ou d'escargot, concentré minéraux vitaminés Autres : Epluchure d'ignames, laitue, mil,

Figure 2 : Types de matières utilisés par les éleveurs du sud-Bénin dans la formulation alimentaire des porcs

Gestion sanitaire et contraintes liées à l'élevage porcin au Sud-Bénin : Le tableau 2 présente les informations liées à la gestion sanitaire et les contraintes liées à l'élevage porcin au Sud- Bénin. On note qu'en matière de soins aux animaux, la plupart des éleveurs (51,43\%) font appel au vétérinaire. Cependant, on remarque que peu d'éleveurs de porcs $(21,43 \%)$ disposent de calendrier prophylactique et l'appliquent. Toutefois, il est à notifier que presque la quasi-totalité des éleveurs dispose de pédiluves hors services. La gale $(68,57 \%)$, la peste porcine africaine $(38,57 \%)$ et la diarrhée $(17,14 \%)$ ont été les pathologies les plus citées. En dehors de ces contraintes sanitaires rencontrées par les éleveurs, d'autres contraintes majeures telles que le manque de moyens financier, la cherté des matières premières, l'indisponibilité de source d'approvisionnement en aliments, les méventes de même que l'incapacité dans la prise en charge des animaux malades par l'administration d'un traitement adéquat ont été enregistrées. 
Tableau 2: Gestion sanitaire et contraintes liées à l'élevage porcin au Sud-Bénin

\begin{tabular}{|c|c|c|}
\hline Variables & $\begin{array}{c}\text { Fréquences } \pm \text { Intervalle de } \\
\text { confiance }(\%) \\
(\mathrm{N}=70)\end{array}$ & Test de Chi carré \\
\hline \multicolumn{3}{|l|}{ Administration des soins } \\
\hline Vétérinaire & $51,43 \pm 11,7$ & \multirow{2}{*}{ NS } \\
\hline Eleveur & $48,57 \pm 11,7$ & \\
\hline \multicolumn{3}{|l|}{ Calendrier prophylactique } \\
\hline Appliqué & $21,43^{\mathrm{b}} \pm 9,6$ & \multirow{2}{*}{ ** } \\
\hline Inexistant & $78,57^{\mathrm{a}} \pm 9,6$ & \\
\hline \multicolumn{3}{|l|}{ Contraintes sanitaires } \\
\hline Gale & $68,57^{a} \pm 10,9$ & \multirow{7}{*}{$* *$} \\
\hline PPA & $38,57^{\mathrm{b}} \pm 11,4$ & \\
\hline Diarrhée & $17,14^{\mathrm{c}} \pm 8,8$ & \\
\hline Parasitose digestive & $7,14^{\mathrm{c}} \pm 6$ & \\
\hline Toux & $5,71^{\mathrm{c}} \pm 5,4$ & \\
\hline Infections (plaie, éruption cutanée, abcès) & $5,71^{\mathrm{c}} \pm 5,4$ & \\
\hline Mammite & $1,43^{\mathrm{c}} \pm 2,8$ & \\
\hline \multicolumn{3}{|l|}{ Autres contraintes } \\
\hline Manque de moyens financier & $100^{a} \pm 0$ & \multirow{6}{*}{$* *$} \\
\hline Cherté des matières premières & $64,29^{\mathrm{b}} \pm 11,2$ & \\
\hline Source d'approvisionnement d'aliment & $32,86^{\mathrm{c}} \pm 11$ & \\
\hline Méventes & $14,29^{\mathrm{d}} \pm 8,2$ & \\
\hline Conflits environnementaux & $11,43^{\mathrm{d}} \pm 7,5$ & \\
\hline Traitement des animaux & $2,86^{\mathrm{d}} \pm 3,9$ & \\
\hline
\end{tabular}

NS : non significatif, ${ }^{*}: \mathrm{P}<0,01 ; \mathrm{N}:$ Nombre d'éleveurs enquêtés ; les fréquences intra classes de la même colonne suivies de lettres $(\mathrm{a}, \mathrm{b})$ différentes présentent une différence significative au seuil de $5 \%$

\subsection{Usages de plantes dans les élevages} porcins du Sud-Bénin: L'enquête réalisée a permis de recenser 42 espèces végétales appartenant à 23 familles botaniques du SudBénin (tableau 3). Ces différentes plantes sont utilisées par les éleveurs pour nourrir (60\%), traiter des affections (19\%) et les deux usages à la fois $(21 \%)$. Moringa oleifera $(15,14 \%)$, Carica papaya $(13,3 \%)$ et Manibot esculenta $(12,39 \%)$ ont été les plantes les plus citées par les éleveurs de porcs. Les parties des plantes les plus utilisées sont les feuilles $(78 \%)$ et les fruits $(12 \%)$ (Figure 3). 


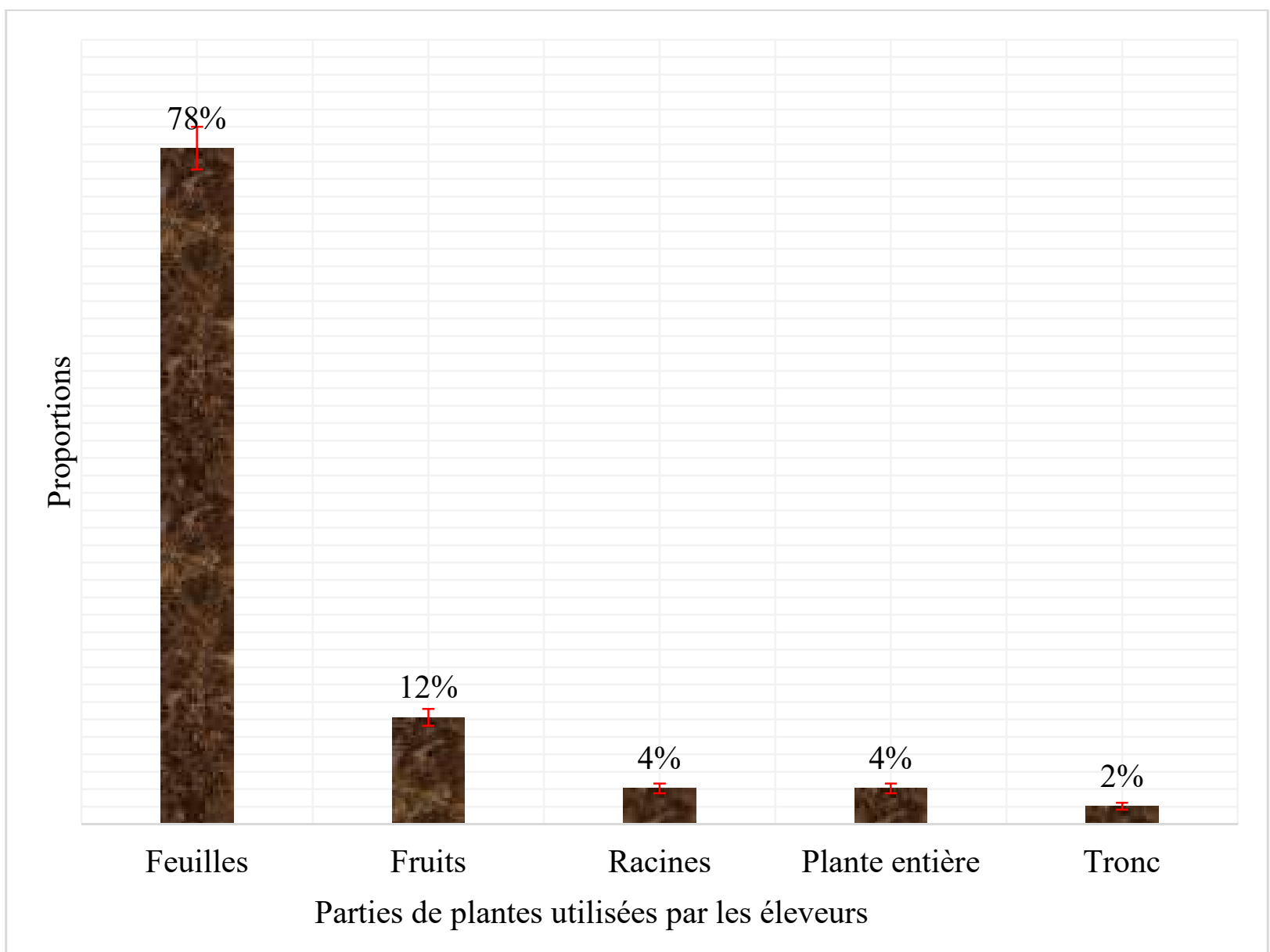

Figure 3 : Parties des plantes utilisées par les éleveurs de porcs du Sud-Bénin

Au total, 22 recettes constituées chacune d'une seule plante et impliquées dans le traitement de 11 pathologies ont été recensées (Tableau 4). Les recettes les plus citées étaient relatives au traitement des parasitoses digestives avec Carica papaya $(17,44 \%)$, l'insuffisance de la sécrétion lactée avec Talinum triangulare (16,28\%), la crise de sixième jour marquée par une diarrhée blanchâtre chez les porcelets avec Moringa oleifera $(11,63 \%)$ et la peste porcine africaine avec Cochlospermum planchonii (6,98\%). Quatre modes de préparation des recettes ont été enregistrés au Sud-Bénin. Il s'agit de la crudité $(74 \%)$, du décocté $(9 \%)$, du pilage $(9 \%)$ et la trituration $(9 \%)$. Les voies d'administrations enregistrées étaient des voies orales $(95 \%)$ et cutanées $(5 \%)$. 
Tableau 3 : Diversité botanique des plantes utilisées dans les élevages de porcs au Bénin selon les éleveurs

\begin{tabular}{|c|c|c|c|c|c|}
\hline Noms scientifiques & $\begin{array}{l}\text { Noms vernaculaires } \\
\text { (Fon, Goun) }\end{array}$ & Familles botaniques & $\begin{array}{c}\text { Parties } \\
\text { utilisées }\end{array}$ & $\begin{array}{c}\text { Buts } \\
\text { d'utilisations }\end{array}$ & $\begin{array}{c}\text { Fréquences de } \\
\text { citations }(\%) \\
(\mathrm{N}=42)\end{array}$ \\
\hline Asystasia gangetica & Yèfè (Fon) & \multirow[t]{4}{*}{ Acanthaceae } & $\mathrm{F}$ & $\mathrm{A}$ & 0,46 \\
\hline Amaranthus bybridus & Fotètè (Fon) & & F & $\mathrm{A}$ & 1,83 \\
\hline Mangifera indica & Manga (Goun) & & $\mathrm{F}, \mathrm{Fr}$ & $\mathrm{A}$ & 0,46 \\
\hline Spondias mombin & $\begin{array}{c}\text { Aklikon (Goun), Djogbema } \\
\text { (Fon) }\end{array}$ & & $\mathrm{F}$ & $\mathrm{A} ; \mathrm{T}$ & 1,83 \\
\hline Elaeis guineensis & Détin (Fon) & Arecaceae & $\mathrm{F}$ & $\mathrm{A}$ & 1,83 \\
\hline Acanthospermum hispidum & Togbaman (Fon) & \multirow[t]{5}{*}{ Asteraceae } & $\mathrm{F}$ & $\mathrm{A}$ & 0,46 \\
\hline Lactuca sativa & Laitue (Fon) & & $\mathrm{F}$ & $\mathrm{A}$ & 0,46 \\
\hline Melanthera scandens & Tohlèssi (Fon, Goun) & & $\mathrm{F}$ & $\mathrm{A}$ & 0,46 \\
\hline Synedrella nodiflora & Hayoé-Hayoé (Goun) & & $\mathrm{Pl}$ & $\mathrm{A}$ & 0,92 \\
\hline Tridax procumbens & Hla dogbo (Fon) & & $\mathrm{F}$ & $\mathrm{A} ; \mathrm{T}$ & 0,92 \\
\hline Cochlospermum planchonii & $\begin{array}{l}\text { Adjinankouvocanfoun } \\
\text { (Fon) }\end{array}$ & Cochlospermaceae & $\mathrm{R}$ & $\mathrm{T}$ & 2,75 \\
\hline Ipomea aquatica & $\begin{array}{l}\text { Towelikan (Goun), } \\
\text { Gbôman (Fon) }\end{array}$ & \multirow[t]{3}{*}{ Convolvulaceae } & $\mathrm{F}$ & $\mathrm{A}$ & 9,17 \\
\hline Ipomea batatas & $\begin{array}{l}\text { Wèli (Goun), Dokwin } \\
\text { (Fon) }\end{array}$ & & $\mathrm{F}, \mathrm{Fr}$ & $\mathrm{A}$ & 1,38 \\
\hline Ipomoea involucrata & $\begin{array}{c}\text { Tôyue (Goun), } \\
\text { Houndranlèn (Goun) }\end{array}$ & & $\mathrm{F}$ & $\mathrm{A}$ & 1,83 \\
\hline Manihot esculenta & Finyiman (Fon) & Euphorbiaceae & $\mathrm{F}, \mathrm{R}$ & $\mathrm{A}$ & 12,39 \\
\hline Acacia sieberiana & Adouwé (Fon) & \multirow[t]{2}{*}{ Fabaceae } & $\mathrm{F}$ & $\mathrm{A}$ & 0,46 \\
\hline Cajanus cajan & Adjayi (Fon) & & $\mathrm{F}$ & $\mathrm{A} ; \mathrm{T}$ & 0,46 \\
\hline
\end{tabular}




\begin{tabular}{|c|c|c|c|c|c|}
\hline Leucena leucocephala & & & $\mathrm{F}$ & A & 0,92 \\
\hline Pterocarpus santalinoides & Gbegbeman (Fon) & & $\mathrm{F}$ & $\mathrm{T}$ & 0,46 \\
\hline Occimum gratissimum & Tchiayo (Fon, Goun) & \multirow[t]{3}{*}{ Lamiaceae } & $\mathrm{F}$ & $\mathrm{T}$ & 0,92 \\
\hline Tectona grandis & Teck (Fon, Goun) & & $\mathrm{F}$ & A & 0,46 \\
\hline Persea americana & Avoka (Fon, Goun) & & $\mathrm{F}$ & A & 0,46 \\
\hline Abelmoschus esculentus & Fevi (Fon) & \multirow[t]{2}{*}{ Malvaceae } & $\mathrm{F}, \mathrm{Fr}$ & $\mathrm{A} ; \mathrm{T}$ & 0,46 \\
\hline Sida linifolia & Djividjivi (Goun) & & $\mathrm{F}$ & A & 0,46 \\
\hline Heterotis rotundifolia & Hèhèman (Goun) & Melastomataceae & $\mathrm{F}$ & $\mathrm{T}$ & 0,92 \\
\hline Azadirachta indica & Kinnininti (Fon, Goun) & Meliaceae & $\mathrm{F}$ & $\mathrm{T}$ & 0,46 \\
\hline Artocarpus altilis & Blèfutu (Fon, Goun) & \multirow[t]{2}{*}{ Moraceae } & $\mathrm{Fr}$ & A & 1,83 \\
\hline Ficus thonningii & $\begin{array}{c}\text { Avlaman (Goun) ; Avla } \\
\text { (Goun) }\end{array}$ & & $\mathrm{F}$ & A & 0,46 \\
\hline Moringa oleifera & $\begin{array}{c}\text { Kpatima (Fon), } \\
\text { Yovokpatin (Goun) }\end{array}$ & Moringaceae & $\mathrm{F}$ & $\mathrm{A} ; \mathrm{T}$ & 15,14 \\
\hline Musa paradisiaca & Kouékoué (Fon) & Musaceae & $\mathrm{F}, \mathrm{Fr}$ & A & 4,13 \\
\hline Psidium guayava & $\begin{array}{l}\text { Kinkoun (Fon, Goun); } \\
\text { Kinkounma (Fon, Goun) }\end{array}$ & Myrtaceae & $\mathrm{F}, \mathrm{Fr}$ & $\mathrm{A} ; \mathrm{T}$ & 0,92 \\
\hline Boerhavia difusa & $\begin{array}{l}\text { Handoukpo (Fon); } \\
\text { Katchuagnin (Fon) } \\
\text { Gbagbada (Goun) }\end{array}$ & Nyctaginaceae & $\mathrm{F}$ & A & 3,21 \\
\hline Petiveria alliacea & Zoroman (Fon) & Phytolaccaceae & $\mathrm{F}$ & $\mathrm{T}$ & 2,29 \\
\hline Zea mays & Gbadé (Fon) & Poaceae & $\mathrm{F}$ & A & 0,92 \\
\hline Talinum triangulare & Glasséma (Fon, Goun) & Portulacaceae & $\mathrm{Pl}$ & $\mathrm{A} ; \mathrm{T}$ & 8,72 \\
\hline Chassalia kolly & Atindjè (Goun) & Rubiaceae & $\mathrm{F}$ & $\mathrm{T}$ & 0,46 \\
\hline Cardiospermum grandiflorium & Togba (Fon) & Sapindaceae & $\mathrm{F}$ & A & 0,92 \\
\hline
\end{tabular}

F : feuilles ; Fr : fruit ; Pl : plante entière ; Tr : tronc ; A : alimentation ; T : traitement ; N : nombre totale de plantes citées 
Tableau 4 : Indications des plantes thérapeutiques dans les maladies ou dysfonctionnement chez le porc selon les éleveurs du Bénin

\begin{tabular}{|c|c|c|c|c|c|c|}
\hline Pathologies & Noms scientifiques & $\begin{array}{c}\text { Parties } \\
\text { utilisées }\end{array}$ & $\begin{array}{l}\text { Mode de } \\
\text { préparation }\end{array}$ & $\begin{array}{c}\text { Voies } \\
\text { d'administrati } \\
\text { on }\end{array}$ & $\begin{array}{l}\text { Durées du } \\
\text { traitement } \\
\quad \text { (jour) }\end{array}$ & Fcr $(\%)$ \\
\hline \multirow{4}{*}{$\begin{array}{l}\text { Crise de } \sigma^{\grave{e}} \text { jour } \\
\text { chez les porcelets } \\
\text { (Diarrhée) }\end{array}$} & Cajanus cajan & $\mathrm{F}$ & Cru & Orale & Jusqu' à guérison & 1,16 \\
\hline & Heterotis rotundifolia & $\mathrm{F}$ & Cru & Orale & Jusqu' à guérison & 2,33 \\
\hline & Moringa oleifera & $\mathrm{F}$ & Cru & Orale & Jusqu' à guérison & 11,63 \\
\hline & Petiveria alliacea & $\mathrm{F}$ & Décoction, Cru & Orale & Jusqu' à guérison & 3,49 \\
\hline \multirow{2}{*}{ Parasitoses internes } & Aqadirachta indica & $\mathrm{F}$ & Cru & Orale & 1 & 2,33 \\
\hline & Carica papaya & $\mathrm{Fr}$ & Cru & Orale & 1 & 17,44 \\
\hline \multirow{4}{*}{ Diarrhée } & Psidium guayava & $\mathrm{F}$ & Cru & Orale & Jusqu' à guérison & 2,33 \\
\hline & Pterocarpus santalinoïdes & $\mathrm{F}$ & Cru & Orale & Jusqu' à guérison & 1,16 \\
\hline & Spondias monbin & $\mathrm{F}$ & Cru & Orale & Jusqu' à guérison & 4,65 \\
\hline & Tridax procumbens & $\mathrm{F}$ & Cru & Orale & Jusqu' à guérison & 2,33 \\
\hline Epistaxis & Spondias monbin & $\mathrm{F}$ & Pilage & Orale & Jusqu' à guérison & 4,65 \\
\hline \multirow{2}{*}{ Inappétence } & Petiveria alliacea & $\mathrm{F}$ & Pilage & Orale & 2 à 3 & 3,49 \\
\hline & Vernonia amygdalina & $\mathrm{F}$ & Trituration & Orale & 2 à 3 & 2,33 \\
\hline \multirow{3}{*}{$\begin{array}{l}\text { Infections (abcès, } \\
\text { éruption cutanées) }\end{array}$} & Abelmoschus esculentus & $\mathrm{F}$ & Cru & Orale & 3 à 7 & 1,16 \\
\hline & Chassalia kolly & $\mathrm{F}$ & Cru & Orale & 3 à 7 & 1,16 \\
\hline & Petiveria alliacea & $\mathrm{F}$ & Cru & Orale & 3 à 7 & 3,49 \\
\hline \multirow{2}{*}{$\begin{array}{l}\text { Insuffisance de } \\
\text { sécrétion lactée }\end{array}$} & Spondias monbin & $\mathrm{F}$ & Cru & Orale & 7 & 4,65 \\
\hline & Talinum triangulare & $\mathrm{F}, \mathrm{Pl}$ & Cru & Orale & 7 & 16,28 \\
\hline $\begin{array}{l}\text { Peste Porcine } \\
\text { africaine }\end{array}$ & $\begin{array}{l}\text { Cochlospermum } \\
\text { planchonii }\end{array}$ & $\mathrm{R}$ & Décoction & Orale & 7 & 6,98 \\
\hline Plaies & Spondias monbin & $\mathrm{F}$ & Trituration & Cutanée & Jusqu' à guérison & 1,16 \\
\hline Retard de chaleur & Occimum gratissimum & $\mathrm{F}$ & Cru & Orale & 2 & 2,33 \\
\hline Anémie & Justicia secunda & $\mathrm{F}$ & Cru & Orale & 7 & 3,49 \\
\hline
\end{tabular}

$\mathrm{F}$ : feuilles ; Fr : fruits ; Pl : plante entière ; R : racines ; Fcr : Fréquence de citation de recette 


\section{DISCUSSION}

\subsection{Caractéristiques} professionnelles des éleveurs et mode d'élevage de porcs au Sud-Bénin: Les données collectées à l'issue de l'enquête montrent que l'élevage des porcs est une activité menée en grande partie par les hommes au Sud du Bénin avec une moyenne d'âge de 44 ans. Néanmoins, certaines femmes s'adonnent à l'activité, ce qui justifie comme dans tout autre domaine d'ailleurs, la non spécificité du sexe par rapport au secteur d'activité. Les mêmes constats ont été rapportés par Kiki et al. (2018) au SudBénin et Ognika et al. (2016) au Congo. Le classement de l'élevage en troisième position après l'agriculture et l'artisanat comme activité principale pourrait s'expliquer par la déscolarisation précoce des enquêtés ou la non obtention d'un diplôme qualifiant pour une formation technique et professionnelle. Ainsi, l'agriculture étant le premier domaine embrassé souvent par les parents, devient un héritage auquel l'élevage de porcs est associé pour valoriser les résidus de récoltes et maintenir une économie au sein de la famille. La priorisation de la race améliorée dans l'élevage de porcs par les éleveurs du Sud du Bénin peut s'expliquer par les avantages des races améliorées telles que la prolificité et la croissance rapide des animaux (Dotché et al, 2019). En effet, au Bénin, les éleveurs de porcs forment des groupements d'association (Govoeyi, 2019) qui bénéficient de formations de mises à niveau par rapport aux différents projets d'amélioration de productivité des porcs (exemple: Projet Recherche Développement-Professionnalisation

et renforcement de la compétitivité de la filière porc par la recherche action en partenariat dans les départements de l'Ouémé et du Plateau au Sud-Est du Bénin, 2015-2020 ). Il ressort de notre étude que les porcs sont élevés en claustration permanente avec une dominance d'habitats en dur. Agbokounou et al. (2016), rapportent que la majorité des éleveurs de porcs sont contraints de pratiquer la claustration permanente ou saisonnière à cause des dégâts causés par la divagation et des mesures prises en son encontre que sont les recommandations et répressions administratives sanitaires, la difficulté de maittrise de l'animal, les conflits entre éleveurs et agriculteurs, l'impact des maladies sur la santé publique, la propagation rapide des maladies. Ceci justifie clairement les résultats enregistrés au cours de notre étude. L'alimentation des porcs au SudBénin est composée par les éleveurs eux-mêmes. Cette composition est faite grâce à l'association de deux ou plusieurs matières premières. Ce système de gestion de l'aliment pourrait être bénéfique aux éleveurs en ce sens que le coût de production n'est plus à chiffrer. Par ailleurs, bien qu'ils disposent de matières premières diversifiées telles que le tourteau de palmiste, le tourteau de coco, le son de maïs, le son de soja, le tourteau de soja, les drêches de brasseries, la farine de moulin, le soja grain, les minéraux avec des compléments d'épluchures et tubercules de manioc de même que les restes de cuisines, ces éleveurs n'arrivent sûrement pas à estimer les besoins des animaux pour espérer les satisfaire. Agbokounou (2001) affirme que dans le cas d'utilisation de ration composée de différents sous-produits, les différentes combinaisons ne répondent à aucune norme précise et se font au hasard selon les disponibilités alimentaires. Cette situation constitue un frein au développement de la filière en ce sens que les techniques de production ne conviennent pas au mode d'élevage et par conséquent la durée de production estimée pour obtenir une croissance donnée chez un animal peut être surpassée ; ce qui pourrait constituer un manque à gagner pour l'éleveur. Kiki et al. (2018) ont rapporté des contraintes d'ordre alimentaire rencontrées par les éleveurs de porcs au cours de leur étude. Ces difficultés étaient principalement l'indisponibilité des matières premières (surtout les sources de protéines qui sont les plus chères) et le coût élevé des matières premières. Les mêmes constats ont été effectués dans notre étude. 
5.1.1 Gestion sanitaire et contraintes liées à l'élevage porcin au Sud-Bénin : En matière de gestion sanitaire, l'enquête réalisée nous révèle que les éleveurs de porcs du sud-Bénin font appel au technicien vétérinaire pour soigner les animaux. Des irrégularités de mesures de biosécurité telles que la disposition et le suivi rigoureux d'un calendrier prophylactique, l'activation des pédiluves à l'entrée des bâtiments d'élevages et la gestion efficiente des cadavres ont été enregistrés. Ces résultats montrent en partie les risques encourus par les éleveurs qui constituent des conditions favorisantes à l'apparition des maladies dans l'élevage. Tout ceci constitue un frein à la production porcine car le non-respect des mesures de biosécurité dans un élevage peut constituer un facteur à la dissémination des germes pathogènes dans l'élevage (Djimenou et al., 2017) et par conséquent dans d'autres. Les pathologies signalées par les éleveurs de porcs au Sud du Bénin sont la gale, la peste porcine africaine (PPA), la diarrhée, les parasitoses digestives, la toux, la mammite, la variole et les infections dues aux éruptions cutanées et abcès. Au nombre de ces maladies, la PPA, les parasitoses digestives et la gale sont les plus fréquentes dans les élevages suivant les indications des éleveurs. La peste porcine africaine demeure une maladie jusque-là sans traitement ni vaccin. Sa déclaration dans une zone constitue une source de problème pour les éleveurs qui bien que conscient de la situation continuent de produire en endémicité. Par ailleurs, Govoeyi et al. (2020) rapporte de nouvelles approches telles que la suspension du jetage des animaux morts dans la brousse ou les rivières, le partage d'informations utiles avec d'autres éleveurs et l'enterrement des porcs morts suivant les normes de biosécurité pour mieux contrôler la situation sanitaire des élevages porcins dans le cas de la PPA. Quant à la gale, plusieurs produits vétérinaires sont disponibles dans les pharmacies vétérinaires, pourtant des plaintes sont toujours enregistrées par rapport à cette maladie récidiviste lorsqu'elle n'est pas bien traitée. La santé animale dépend de l'équilibre entre, d'une part, la présence d'agents infectieux (pression d'infection) et d'autre part, la protection et la résistance de l'hôte (immunité innée et adaptative, vaccination, stress, etc.), cet équilibre étant fortement influencé par l'environnement de l'animal et de l'élevage (biosécurité, logement, alimentation, etc.) (Tona-Tona et al., 2019). Outre les problèmes d'ordres pathologiques qui handicapent l'élevage de porcs au Sud du Bénin, les éleveurs ont notifié d'autres contraintes liées à l'élevage de porcs. Ces contraintes prennent en compte tous les aspects de l'élevage de porcs. Il s'agit du manque de moyens financiers, première contrainte majeure suivie de celle sanitaire; l'indisponibilité de sources d'approvisionnement d'aliments ou intrants couplée avec la cherté de ces derniers. Ces difficultés freinent l'élevage de porcs, décourageant ainsi les éleveurs du domaine ayant pour conséquence directe l'abandon de l'élevage de porcs au profit d'autres activités comme l'ont rapporté Djimènou et al. (2017). De plus, lorsqu'ils parviennent à produire face à ces contraintes sus citées, ils sont soumis parfois à des méventes dues en partie à l'importation du porc depuis le Nigéria, mais aussi à des conflits environnementaux du fait du manque d'entretien des porcheries et de l'organisation efficiente de la gestion des effluents.

\subsubsection{Usages de plantes dans les élevages} porcins du Sud-Bénin : L'usage des plantes a toujours été partie intégrante dans les systèmes de productions animales surtout traditionnels. La présente étude nous a permis de recenser 42 plantes appartenant à 23 familles botaniques. Les résultats obtenus montrent la multiplicité des ressources végétales dont regorge la flore béninoise en relation avec les connaissances des éleveurs. Ces plantes sont utilisées pour nourrir et soigner les animaux.. Les buts d'utilisations des plantes enregistrés dans la présente étude justifient l'importance et l'implication des plantes dans la gestion alimentaire et sanitaire des élevages porcins. Non seulement, ces plantes pourrait constituer des alternatives aux produits pharmaceutiques mais participeraient ainsi à la réduction du coût de production dans l'élevage. 
L'enregistrement des feuilles et des fruits comme étant les parties des plantes les plus utilisées peut s'expliquer par la facilité de récolte et la disponibilité de ces organes. Des études menées au Bénin (Agbokounou, 2001; Nonfon, 2005; Kiki et al., 2018), au Tchad (Logtene and Koussou, 2003) et en République Démocratique du Congo (Kambashi et al., 2010) ont révélé aussi l'utilisation de Manihot esculenta, Ipomea batatas, Ipomea aquatica, Ipomea involucrata, Amaranthus bybridus, Carica papaya, Talinum triangulare, Synedrella nodiflora, Tridax procumbens, Lactuca taraxaxifolia, Commelina bengalensis, Acanthospermum bispidum, Panicum maximum, Cajanus cajan, Leucaena leucocephala, Moringa oleifera, Mucuna pruriens dans l'alimentation des porcs. Ces résultats sont partiellement similaires à ceux obtenus au cours de notre étude. En effet, compte tenu de la richesse en protéine digestible de certaines de ces espèces (Kambashi et al., 2010), les plantes peuvent être utilisés dans l'alimentation des porcs sous forme de fourrage dans les conditions de rareté des ressources riches en protéines et leur limite d'incorporation dans la ration de ces animaux peut aller à $20 \%$ (Kambashi et al., 2014) . Il faut noter que les résultats de notre étude rapportent l'utilisation des plantes comme fourrage directement servie aux animaux. Cette technique pourrait être améliorée par l'incorporation directe des plantes dans l'aliment des animaux. Une perspective de recherche s'ouvre dans ce cas sur l'effet des fourrages servis directement aux porcs en comparaison aux fourrages incorporés dans l'alimentation de base des porcs. En général, les fourrages sont très riches en fibres alimentaires qui favoriseraient le transit intestinal et limiteraient des pertes en azote lors du stockage des lisiers (Agbokounou et al., 2016). Par contre, ils contiendraient certains facteurs antinutritionnels qui limiteraient l'efficacité de leur utilisation. Certains auteurs rapportent aussi que les fruits servis aux animaux sont pourris. C'est le cas de la papaye et des mangues avariées souvent utilisées au Bénin (Nonfon, 2005), les pastèques et les fruits sauvages des arbres de Adansonia digitata et Ziziphus mauritiana surtout servis aux animaux au Zimbabwe (Mushandu et al., 2005) et la pomme d'acajou puis des mangues avariées en Basse Casamance au Sénégal (Missohou et al., 2001). Par ailleurs, l'intérêt des fourrages et fruits réside aussi dans l'apport de vitamines, de minéraux et certaines de ces espèces auraient des propriétés médicinales (Agbokounou, 2001). Au cours de notre étude, la crise de $6^{\text {ème }}$ jour chez les porcelets (diarrhée blanchâtre au sixième jour de naissance des porcelets), la parasitose interne, la diarrhée, l'épistaxis, l'inappétence, la PPA, le retard de chaleur, l'anémie, la mammite et les infections dues aux abcès et éruption cutanée ont été les maladies contre lesquelles des indications thérapeutiques à base de plante ont été enregistrées. Certaines plantes identifiées au cours de notre étude ont été indiquées dans le traitement d'autres maladies animales. C'est le cas de Azadirachta indica, Parkia biglobosa, Mangifera indica et Carica papaya utilisées respectivement pour le traitement des ectoparasites, la météorisation, la pasteurellose bovine et la rétention placentaire (Dassou et al., 2014) ; Manihot esculenta, Mangifera indica et Elaeis guineensis utilisées respectivement dans le traitement de la fièvre aphteuse, la maladie de Newcastle et la variole aviaire (Kpodékon et al., 2015). De même Houndje et al. (2016), ont rapporté Parkia biglobosa et Boerhavia diffusa comme étant utilisées par les éleveurs dans le traitement de la fièvre aphteuse. Ces différents résultats confirment la multiplicité des propriétés pharmacologiques dont les plantes sont douées. Par ailleurs, il faut noter que la peste porcine africaine, maladie virale hautement contagieuse et sévissant dans presque tous les continents constitue à ce jour un frein pour maximiser la production porcine. Dans la présente étude, Cochlospermum planchonii a été rapporté par certains éleveurs de porcs du SudBénin comme étant utilisée dans le traitement de la PPA. Cette même plante a été citée par les éleveurs de porcs pour la même maladie au cours de l'étude réalisée par Kpodékon et al. (2015) au Bénin sur les pathologies virales dominantes des élevages extensifs et semi-intensifs et leurs 
modes de traitement en ethnomédecine vétérinaire. En plus de cette plante, Cissus quadrangularis, Adansonia digitata, Momordica charantia, Datura metel, Cussonia arbórea et Ageratum conyzoides ont été rapportés par les mêmes auteurs dans le traitement de la PPA. Au vu de cette diversité floristique soulignée par les éleveurs de porcs, il s'avère nécessaire de mener des études plus poussées dans la recherche d'une alternative endogène parallèlement aux thérapies vaccinales

\section{CONCLUSION}

Cette étude réalisée renseigne sur les pratiques d'élevage de porcs au Sud-Bénin. Les différents échanges avec les éleveurs leur permettront de mieux revoir leurs méthodes d'élevages surtout en matière de biosécurité. En matière d'utilisation de plantes pour la gestion alimentaire des porcins, de nouvelles propositions de formules alimentaires incorporant les plantes à propriétés nutritives pourront être mises à disposition des éleveurs.

\section{ACKNOWLEGMENT}

Nous remercions les agents techniques de l'Agence Territoriale du Développement Agricole Pôle 7 qui ont facilité la réalisation de l'étude de terrain. Nos remerciements vont aussi à l'endroit du Ministère de l'Enseignement

\section{REFERENCES}

Adjanohoun EJ, Adjakidje V, Ahyi MRA, Ake Assi L, Akoegninou A, d'Almeia J, Apovo F, Boukef K, Chadare M, Cusset G, Dramane K, Eymé J, Gassita JN, Gbaguidi M, Goudote E, Guinko S, Houngnon P, Issa L, Kéita A, Kiniffo HV, Koné-Bamba D, Musampa Nseyya A, Saadou M, Sodogandgi T, de Souza S, Tchabi A, Zinsou Dossa C, Zohoun T: 1989. Contribution aux études ethnobotaniques et floristique en République Populaire du Bénin Collection "Médecine traditionnelle et pharmacopée". Agence de Coopération Culturelle et Technique 111, 339-405. en cours de développement contre la PPA. La voie d'administration orale des recettes médicinales est dominante suivant notre étude avec les modes de préparation de crudité et de décocté. Les résultats obtenus peuvent s'expliquer par la nature des maladies à traiter et l'espèce en cause. Il est surtout important de noter que le mode de préparation ainsi que les parties à utiliser de la plante dépendent du type de pathologies à traiter (Houndje et al., 2016).

Quant à la gestion sanitaire, plusieurs Médicaments Traditionnelles Améliorés (MTA) pourront ressortir après vérification et formulation à travers les recettes fournies par les éleveurs et mises à disponibilité. Ainsi, les informations recueillies à l'issue de cette étude, serviront aux acteurs chargés de la gestion de la filière porcine au Bénin pour booster la production porcine.

Supérieur et de la Recherche Scientifique qui à travers son Programme «Appui aux Doctorant » (PAD-2018) nous a permis de mener les activités dans de bonnes conditions.

Agbokounou AM: 2001. Etude des besoins énergétiques et protéiques du porc local béninois en phase de démarragecroissance. Mémoire de DEA en Zootechnie, Faculté universitaire des sciences agronomiques de Gembloux, Belgique 92p.

Agbokounou AM, Ahounou GS, Youssao AKI, Mensah GA, Koutinhouin B. and Hornick J-L : 2016. Caractéristiques de l'élevage du porc local d'Afrique. Journal of Animal \&Plant Sciences 30:4701-4713.

Akoègninou A, Van der Burg WJ. and Van der Maesen LJG: 2006. Flore analytique du Bénin, Backhuys Publishers, Wageningen; 1034p 
Codjo AB : 2003. Estimation des besoins énergétiques du porc local du Bénin en croissance entre 7 et $22 \mathrm{~kg}$ de poids vif. Tropicultura 21:56-60.

Dassou HG, Ogni CA, Yédomonhan H, Adomou AC, Tossou M, Dougnon JT. and Akoègninou A : 2014. Diversité, usages vétérinaires et vulnérabilité des plantes médicinales au Nord-Bénin. International Journal of Biological and Chemical Sciences 8:189-210.

Djimenou D, Adoukonou-Sagbadja H, Koudande OD, Chrysostome C, Hounzangbe-Adote S, and Agbangla C: 2017. Characteristics and constraints of pigs breeding in Sub-humid zone of Benin. Int J Curr Res Biosci Plant Biol in Biosciences and Plant Biology. 4:38-49.

Dotché IO, Ahounou SG, Salifou CFA, Biobou R, Kiki P, Govoeyi B, AntoineMoussiaux N, Dehoux J-P, Mensah GA. and Farougou $S$ : 2018. Critères de choix et de réforme des reproducteurs mâles et femelles dans les élevages de porcs des départements de l'Ouémé et du Plateau au Bénin. Revue d'élevage et de médecine vétérinaire des pays tropicaux $12 \mathrm{p}$

Dotché IO, Bankolé CB, Dahouda M, Biobou R, Bonou GA, Antoine-Moussiaux N, Dehoux J-P, Thilmant P, Mensah GA, and Koutinhouin BG : 2019. Comparison of reproductive performances of local and improved pigs reared in south Benin. Tropical animal bealth and production 1-12.

Eklu-Natey RD. and Balet A: 2012. Pharmacopée africaine. Dictionnaire et monographies multilingues du potentiel médicinal des plantes africaines: Afrique de l'ouest. Volume 1, Lausanne : Editions d'en-bas; Genève : Traditions et Médecine, 912p.

FAO : 2012. Secteur Porcine République Démocratique du Congo. Revues nationales de l'élevage de la division de la production et de la santé animales de la FAO. No. 2. Rome 85p.
FAOSTAT-FAO : Equilibres des produits Elevage et pêche - Equivalent primaire. 2019 ;

www.fao.org/faostat/fr/\#data/bl/visu alize (consulté le 09 décembre 2019)

FAOSTAT-FAO : Equilibres des produits Elevage et pêche - Equivalent primaire. 2020 ; www.fao.org/faostat/fr/\#data/QA (consulté le 26 avril 2020)

Govoeyi B, Ahounou SG, Agbokounou AM, Salifou CF, Dotche IO, Kiki PS, Karim IYA, and Antoine-Moussiaux N: 2019. Participatory innovation analysis along livestock value chains: Case of swine value chain in Benin. Agricultural systems 174:11-22, Elsevier

Govoeyi B, Agbokounou AM, Camara Y, Ahounou SG, Dotche IO, Kiki PS, Karim IYA, Delabouglise A, and Antoine-Moussiaux N: 2020. Social network analysis of practice adoption facing outbreaks of African Swine Fever. Preventive Veterinary Medicine 105008, Elsevier.

Hedji CC, Houinato M, Houndonougbo F. and Fiogbe E: 2015. Assainissement de l'environnement par la valorisation des ressources non conventionnelles en alimentation de porcs en croissance. International Journal of Biological and Chemical Sciences 9:1929-1936.

Houndje EMB, Ogni CA, Noudeke N, Farougou S, Youssao AKI. and Kpodekon TM: 2016. Recettes ethnovétérinaire à base de plantes médicinales utilisées pour le traitement de la fièvre aphteuse au Bénin. International Journal of Biological and Chemical Sciences 10:20902107.

INSAE: 2015. Que retenir des effectifs de population en 2013. INSAE, ministère du Développement, de l'Analyse, économique et de la Prospective, Bénin, $33 \mathrm{p}$.

Kambashi B, Boudry C, Picron P, Kiatoko H, Thewis A. and Bindelle J : 2010. La 
valorisation des aliments non conventionnels dans les systèmes d'élevage porcin en RDC. Colloques et congrès scientifiques du 19 Octobre 2010, Université de Liège, Gembloux Agro-Bio Tech, https://orbi.uliege.be/handle/2268/74 222 consulté le 9/03/2020

Kambashi B, Picron P, Boudry C, Thewis A, Kiatoko H. and Bindelle J: 2014. Nutritive value of tropical forage plants fed to pigs in the Western provinces of the Democratic Republic of the Congo. Animal Feed Science and Technology 191:4756.

Kiki PS, Dahouda M, Toleba SS, Ahounou SG, Dotché IO, Govoeyi B, AntoineMoussiaux N, Mensah GA, Farougou S. and Karim IYA: 2018. Gestion de l'alimentation des porcs et contraintes de l'élevage porcin au Sud-Bénin. Revue d'élevage et de médecine vétérinaire des pays tropicaux 71: 8p

Koudouvo K, Karou DS, Kokou K, Essien K, Aklikokou K, Glitho IA, Simpore J, Sanogo R, De Souza C. and Gbeassor M: 2011. An ethnobotanical study of antimalarial plants in Togo Maritime Region. Journal of ethnopharmacology 134:183-190.

Kpodékon TM, Ogni CA, Dassou H, Dougnon TJ, Boko C, Koutinhouin GB, Goussanou JSE, Akoegninou A. and Youssao I: 2015. Dominant viral pathologies in the extensive and semiintensive animal breeding and their treatment mode in ethno veterinary medicine in Benin. Veterinary world 8:1424.

Logtene YM. and Koussou MO : 2003. L'élevage porcin, un élevage ignoré, mais pourtant bien implanté dans les agrosystèmes ruraux et périurbains du Tchad. In Savanes africaines: des espaces en mutation, des acteurs face à de nouveaux défis. Actes du colloque, Garoua, Cameroun, Cirad, Prasac 9p
Missohou A, Niang M, Foucher H. and Dieye PN: 2001. Pig production systems in Lower Casamance (Senegal). Cahiers d'Etudes et de Recherches Francophones Agricultures (France) 10 : 405-408

Mushandu J, Chimonyo M, Dzama K, Makuza SM. and Mhlanga FN : 2005. Influence of sorghum inclusion level on performance of growing local Mukota, Large White and their F1 crossbred pigs in Zimbabwe. Animal feed science and technology 122:321-329.

Nonfon WR : 2005. La filière de production du porc local au Bénin : l'amélioration de sa productivité par l'alimentation. Thèse de Doctorat en Sciences agronomiques et Ingénierie biologique, Faculté Universitaire des Sciences Agronomiques de Gembloux, Belgique, 236p.

Ognika AJ, Mopoundza P, Okandza Y. and Akouango P : 2016. Adaptation productive et reproductive des porcs large white élevés en race à Brazzaville (république du Congo). Journal of Animal \&Plant Sciences 30:4727-4735.

Tona-Tona A, Thilmant P, Ntoto-M'vubu R. and Okitayela-Onawoma F: 2019. Appréciation du niveau de biosécurité des élevages porcins périurbains de Kinshasa, République Démocratique du Congo (RDC). Afrique Science 15:197210.

Youssao AI, Koutinhouin GB, Kpodekon TM, Bonou AG, Adjakpa A, Dotcho CDG. and Atodjinou FTR : 2008a. Production porcine et ressources génétiques locales en zone périurbaine de Cotonou et d'Abomey-Calavi au Bénin. Revue d'Élevage et de Médecine Vétérinaire des Pays tropicaux 61:235-243.

Youssao AKI, Kpodékon TM, Koutinhouin GB, Adjapka A, Yacoubou A. and Ahounou S: 2008b. Influence de la castration des mâles sur les performances de croissance, les 
caractéristiques de la carcasse et les qualités de la viande du porc local du Bénin. Bull Rech Agron Bénin 61:1-24.

Youssao AKI, Koutinhouin GB, Kpodekon TM, Yacoubou A, Bonou AG, Adjakpa A, Ahounou S. and Taiwo R: 2009. Amélioration génétique des performances zootechniques du porc local du Bénin par croisement avec le Large White. International Journal of Biological and Chemical Sciences 3 : 653-662 